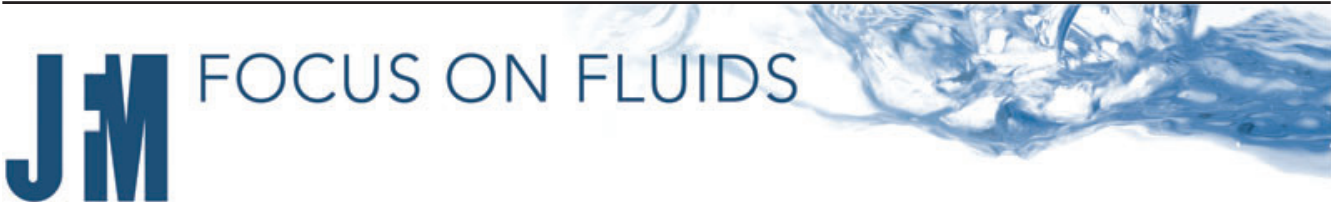

\section{Predicting evaporation rates of droplet arrays}

\author{
David J. Fairhurst $\dagger$ \\ Department of Physics and Mathematics, Nottingham Trent University, Clifton Lane, Nottingham \\ NG11 8NS, UK
}

The evaporation of multiple sessile droplets is both scientifically interesting and practically important, occurring in many natural and industrial applications. Although there are simple analytic expressions to predict evaporation rates of single droplets, there are no such frameworks for general configurations of droplets of arbitrary size, contact angle or spacing. However, a recent theoretical contribution by Masoud, Howell \& Stone (J. Fluid Mech., vol. 927, 2021, R4) shows how considerable insight can be gained into the evaporation of arbitrary configurations of droplets without having either to obtain the solution for the concentration of vapour in the atmosphere or to perform direct numerical simulations of the full problem. The theoretical predictions show excellent agreement with simulations for all configurations, only deviating by $25 \%$ for the most confined droplets.

Key words: drops, condensation/evaporation, contact lines

\section{Introduction}

Sweat evaporating from an athlete's skin, agrochemicals sprayed onto crops, inkjet printers, industrial spray coolers and virus transmission from infected surfaces all depend on the collective evaporation of many droplets on a surface. However, despite these and many other applications, nearly all of the considerable analytical, experimental and numerical work on droplet evaporation has focused on a single droplet. Typically, the rate of evaporation is controlled by the diffusion of vapour in the quiescent atmosphere, and is therefore described by the 'diffusion-limited model'. In its simplest form this model involves solving Laplace's equation for the concentration of vapour in the atmosphere subject to mixed boundary conditions representing complete saturation at the free surface of the droplet, no flux of vapour through the unwetted part of the substrate and a far-field condition representing the ambient vapour concentration. Lebedev (1965) and Popov (2005) provide a well-known analytic solution to this problem, giving a simple form for the diffusive vapour field and evaporation rate for a single droplet.

However, in practice, droplets rarely occur in isolation, and so understanding the interactions between multiple droplets is of considerable scientific and practical importance. Although research on three-dimensional arrays of droplets (e.g.

$\dagger$ Email address for correspondence: david.fairhurst@ntu.ac.uk 


\section{D.J. Fairhurst}

aerosols) is an extensive field, two-dimensional arrays of interacting sessile droplets on a surface are much less studied, and we summarise the key findings below. Kokalj et al. (2010) applied computational methods to droplet arrays and demonstrated that cooling was greatest for small dense droplet arrays, which would lead to a reduction in evaporation rate. Sokuler et al. (2010) show that, in contrast to isolated droplets with constant contact angle, for which the evaporation rate (defined as the change in droplet volume $V$ per unit time $t$ ) reduces over time as $J=\mathrm{d} V / \mathrm{d} t \propto t^{1 / 2}$, for large droplet arrays, approximated as a continuous film, the evaporation rate is constant over time. For droplets with a pinned contact line, Carrier et al. (2016) also find, both experimentally and theoretically, that the total evaporation rate depends on droplet size and configurations. For small droplets, evaporation is diffusion limited and proportional to the droplet's size, whereas for droplets larger than around $20 \mathrm{~mm}$, evaporation becomes convective with the rate proportional to the droplet area. They introduce the idea of a 'superdrop' to predict the evaporation rate of droplet arrays and give a simple analytic expression to describe how the evaporation is hindered due to the presence of other droplets. For droplets dissolving in a surrounding fluid (an analogous situation also described by the Laplace equation), Chong et al. (2020) found evidence for a similar transition from diffusion to a convective plume. However, in their case, convection in the more dense arrays led to a surprising increase in dissolution rate.

A key concept throughout these studies is the so-called 'shielding' effect, in which the presence of vapour from the other droplets reduces the evaporation rate (and hence increases the lifetime) of a droplet relative to that of the same droplet in isolation. However, none of the works mentioned above explicitly consider the variation in evaporation rate from one droplet to another, which will depend strongly on each droplet's position within the array. This more involved problem was in fact first solved by Fabrikant (1985) for potential flow through a perforated membrane. Although a seemingly unrelated problem, Wray, Duffy \& Wilson (2020) recognised it as being analogous to the evaporation of zero-thickness circular droplets, and were able to integrate the expression for evaporation rate to obtain droplet drying times. These results are formally valid in the asymptotic limit where the droplets are well separated, with the problem reducing to a system of $N$ linear equations describing the evaporation rate from each droplet. Both Fabrikant (1985) and Wray et al. (2020) found good agreement between the theoretical predictions for a pair of identical droplets with those of numerical calculations right up to the limit of touching droplets. In this case the effect of shielding increases the lifetime of the droplets by one third. In addition Wray et al. (2020) obtained expressions describing the variation of flux across the surface of each droplet.

Very recently, Edwards et al. (2021) found very good agreement between the theoretical predictions of Fabrikant (1985) and experimental results obtained using an interferometric technique to directly measure the individual evaporation rate of up to 25 droplets in ten different configurations.

\section{Overview of Masoud, Howell \& Stone (2021)}

The work of Masoud et al. (2021) is important and novel as it extends the findings of Fabrikant (1985), removing the restrictions of thin droplets and circular contact lines. They used Green's second identity to simply and elegantly obtain an exact relationship between the local flux and total evaporation rate from the droplets. Using the method of reflections and assuming that the droplets are well separated, they obtained a system of $N$ linear equations for the evaporation rates from each droplet $J_{n}$ involving only the rate for the isolated droplet $\hat{J}_{n}$ and $\phi\left(r_{n m}\right)$, the normalised vapour concentration at the location of the 


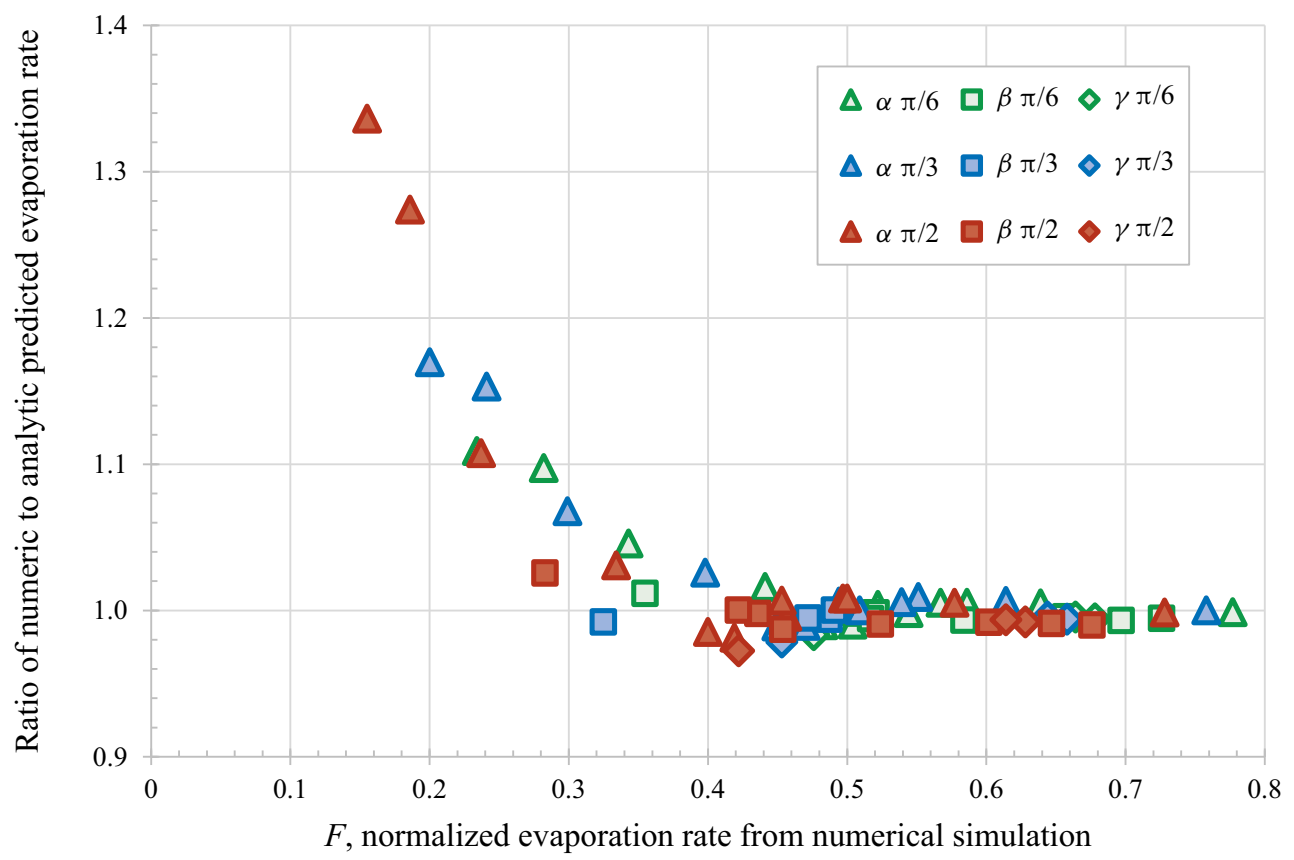

Figure 1. Comparison of the predictions using the new analytical approach to direct numerical simulations, for various droplet arrangements and confinements $(\alpha-$ most confined, $\beta$ and $\gamma-$ least confined) and initial contact angles $(\pi / 6, \pi / 3$ and $\pi / 2)$ for droplet separation of 2.5 radii.

$m$ th droplet

$$
F=\frac{J_{n}}{\hat{J}_{n}}=1-\sum_{m=1}^{N} \phi\left(r_{n m}\right) \frac{J_{m}}{\hat{J}_{n}} .
$$

Using for example the expression of Popov (2005) for $\phi\left(r_{n m}\right)$, this set of $N$ equations can be solved giving the evaporation rates for each droplet. For low contact angles the vapour concentration term reduces to $\phi\left(r_{n m}\right)=(2 / \pi) \arcsin \left(a_{n} / r_{n m}\right)$, where $a_{n}$ is the radius of the $n$th droplet recovering the simpler form derived by Fabrikant (1985).

The authors evaluated the accuracy of their theoretical predictions by comparing with the results of direct numerical simulations for twelve different configurations of identical spherical-cap droplets using three different contact angles $(\pi / 6, \pi / 3$ and $\pi / 2)$ and droplet separations of 2.5 and 3 radii (i.e. 72 different calculations). Figure 1 replots the dataset provided in table 1 of Masoud et al. (2021) for the closest separations, confirming the excellent agreement for the faster evaporation droplets, with $F>0.4$. For slower evaporating droplets $(F<0.4)$ which are more confined and have larger contact angles, the theoretical results are systematically slower than the numerical results.

\section{Future}

The great merit of this work is that it quantifies the significance of the shielding effect in arbitrary configurations of droplets of different sizes and contact angles without having either to obtain the solution for the concentration of vapour in the atmosphere or to perform time-consuming and technically challenging direct numerical simulations. Moreover, it 


\section{D.J. Fairhurst}

opens the door to a greater understanding of the many applications of this effect and could lead to improvements in inkjet printers or cooling systems, for example.

Here, we briefly mention three specific directions for potential future work. Firstly, the systematic discrepancy seen for the most confined droplets could be investigated further. As the droplets have a centre to centre separation of 2.5 radii the theory is expected to be accurate, so the disagreement is most likely due to a more subtle effect of confinement rather than the prediction being applied beyond its valid range. Any improvement to the theory should be verified against additional numerical and experimental work. Secondly, it would be interesting to explore the collective behaviour of an increasing number of droplets and thereby determine to what extent a collection of many droplets can be considered as one large 'super droplet' of an appropriate shape and size, as discussed by Carrier et al. (2016). Thirdly, and most generally, the same theoretical approach can be applied to other physical situations governed by Laplace's equation such as for example the dissolution of microbubbles, as reviewed by Lohse \& Zhang (2015) and Qian, Arends \& Zhang (2019).

Declaration of interests. The author reports no conflict of interest.

\section{Author ORCIDs.}

() David J. Fairhurst https://orcid.org/0000-0001-5311-0762.

\section{REFERENCES}

Carrier, O., Shahidzadeh-Bonn, N., Zargar, R., Aytouna, M., Habibi, M., Eggers, J. \& Bonn, D. 2016 Evaporation of water: evaporation rate and collective effects. J. Fluid Mech. 798, 774-786.

Chong, K.L., LI, Y., NG, C.S., Verzicco, R. \& LohSE, D. 2020 Convection-dominated dissolution for single and multiple immersed sessile droplets. J. Fluid Mech. 892, A21.

Edwards, A.M.J., Cater, J., Kilbride, J.J., Le Minter, P., Brown, C.V., Fairhurst, D.J. \& OUALI, F.F. 2021 Interferometric measurement of co-operative evaporation in 2D droplet arrays. Appl. Phys. Lett. 119, 151601.

FABRIKant, V.I. 1985 On the potential flow through membranes. Z. Angew. Math. Phys. 36 (4), 616-623.

KoKAlJ, T., Cho, H., JenKo, M. \& LeE, L.P. 2010 Biologically inspired porous cooling membrane using arrayed-droplets evaporation. Appl. Phys. Lett. 96 (16), 163703.

LeBedEV, N.N. 1965 Special Functions and their Applications. Prentice-Hall Inc.

LoHSE, D. \& ZHANG, X. 2015 Surface nanobubbles and nanodroplets. Rev. Mod. Phys. 87, 981-1035.

Masoud, H., Howell, P.D. \& Stone, H.A. 2021 Evaporation of multiple droplets. J. Fluid Mech. 927, R4.

Popov, Y.O. 2005 Evaporative deposition patterns: spatial dimensions of the deposit. Phys. Rev. E 71 (3), 036313.

QIAN, J., ARENDS, G.F. \& ZHANG, X. 2019 Surface nanodroplets: formation, dissolution, and applications. Langmuir 35, 12583-12596.

Sokuler, M., Auernhammer, G.K., Liu, C.J., Bonaccurso, E. \& Butt, H.-J. 2010 Dynamics of condensation and evaporation: effect of inter-drop spacing. Europhys. Lett. 89 (3), 36004.

Wray, A.W., DufFY, B.R. \& Wilson, S.K. 2020 Competitive evaporation of multiple sessile droplets. J. Fluid Mech. 884, A45. 